\title{
THE SPATIAL EXTENT OF ATTENTION DURING DRIVING
}

\author{
George J. Andersen, Rui Ni \\ Department of Psychology \\ University of California Riverside \\ Riverside, California, USA \\ E-mail: Andersen@ucr.edu \\ E-mail: ruini@ucr.edu
}

\begin{abstract}
Summary: The present study examined the limits of spatial attention during driving using a dual-task performance paradigm. Drivers were asked to follow a lead vehicle that varied in speed while also detecting a light change in an array located above the roadway. Reaction time increased and accuracy decreased as a function of the horizontal location of the light change and the distance, from the driver, of the light change. In addition, RMS error in car following increased immediately following the light change. These results demonstrate that when drivers attend to a centrally located task, their ability to respond to other events varies as a function of horizontal visual angle and distance in the scene.
\end{abstract}

\section{INTRODUCTION}

Previous research has shown that human observers have a limited ability to attend to the entire visual scene. In the context of driving this limitation, which has been referred to as the useful field of view (UFOV; Ball et al., 1993), has been measured by requiring observers to perform two tasks - one task based on responding to visual information present in the central visual field with a secondary task based on responding to information present in more peripheral regions of the visual field. Performance on the UFOV has been shown to be a good predictor of accident risk in driving (Sims et al., 2000; Owsley et al., 1991; 1998). The assessment of the UFOV is performed by requiring subjects to make responses to a $2 \mathrm{D}$ array of information on a computer screen. In contrast, driving involves processing and responding to information within a 3D world. Previous research has demonstrated that we have a limited ability to attend to information in depth (Andersen, 1990; Andersen and Kramer 1993; Atchley et al., 1997). The purpose of the present study was twofold: first, to assess changes in the spatial extent of attention using driving relevant tasks and second, to measure changes in the spatial extent of attention as a function of the 3D distance of the secondary task.

Driving simulation displays were generated that depicted a roadway scene of city blocks with traffic in two adjacent lanes and a vehicle located directly in front of the driver's vehicle. The two tasks examined were car following and detecting a light change in an array of lights located above the roadway. For the car following task, drivers were shown an initial period of travelling at a constant speed and fixed distance behind the lead vehicle. After the initial period, the lead vehicle varied speed and the drivers were asked to maintain the initial driving distance. While performing the car following task the driver's vehicle would pass under an array of lights located above the roadway. At prespecified distances, one of the lights would change and drivers were asked to indicate whether the light change occurred on the left or right side of the array. To measure the spatial extent of attention, we systematically varied the horizontal position of the light change. The array consisted of 21 lights with 10 lights located on the right and left of the 
light alligned with the center of the driver's position. The light change occurred at either the 3,6 or 9 light locations from the center (0 position) light and occurred on either the right or left side.

\section{EXPERIMENT}

\section{Methods}

Drivers. The drivers were 6 college-age students at the University of California, Riverside, who were paid for their participation. All drivers had a minimum of 2 years of driving experience, had normal or corrected to normal vision, and were naïve to the purpose of the experiment.

Design. The independent variables were the distance of the light when a change occurred $(48,72,96$, or $120 \mathrm{~m}$ ), the horizontal position of the light when a change occurred (3, 6 or 9 position), and the side of the light change (left or right). All other variables were run as withinsubject variables.

Apparatus. The displays were presented on a Dell PC computer system. The visual angle of the displays was $47 \mathrm{deg}$ by $26 \mathrm{deg}$, with the refresh rate at $60 \mathrm{~Hz}$ and the resolution at 1024 by 768 . The display was $25.8 \mathrm{deg}$ by $34.7 \mathrm{deg}$. Display update was $60 \mathrm{~Hz}$. A

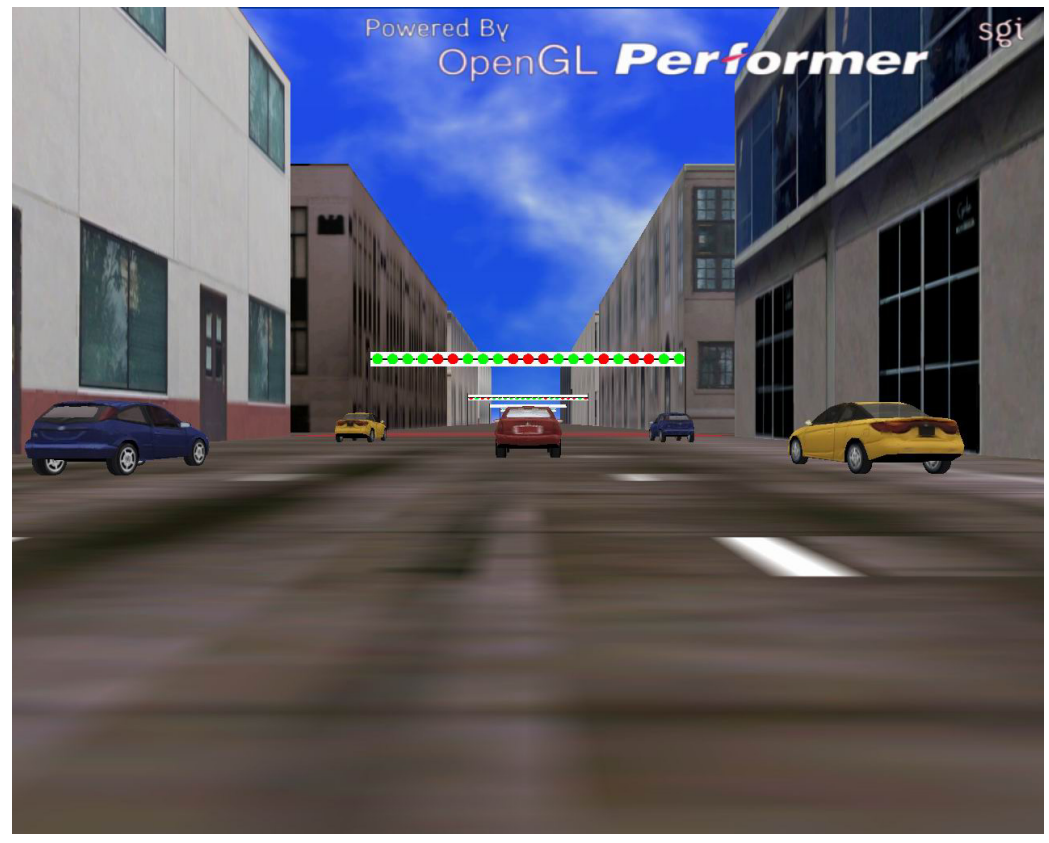

Figure 1. Front view of the driving scene used in the present study. Thrustmaster Formula T2 control system, including acceleration and brake pedals, was used for closed loop control of the simulator. The foot pedal and a BG systems cereal box were used to produce closed-loop control that was updated at $36 \mathrm{~Hz}$. Drivers viewed the displays binocularly at a distance of approximately $40 \mathrm{~cm}$ from the screen.

Stimuli. The displays depicted a roadway scene of city blocks (see Figure 1). Located in front of the driver was a lead vehicle that varied speed. The roadway consisted of black and white gravel texture pattern to simulate asphalt. A double yellow line was present to indicate the traffic lanes. The city buildings and lead vehicle were produced by digitally photographing real buildings and vehicles and using the digital images as texture maps for the roadway scenes. The images were digitally altered to increase the realism of the simulator scene (e.g., remove specular highlights, add shading). Lane width was $3.8 \mathrm{~m}$. Drivers were presented with a car-following scenario in which the lead vehicle varied its velocity according to a sum of 3 equal-energy sinusoids (i.e., the peak accelerations and decelerations of each sine wave in the signal were equivalent). The three frequencies used were $.033, .083$, and $.117 \mathrm{~Hz}$. The corresponding amplitudes for these sinusoids were: $9.722,3.889$, and $2.778 \mathrm{kph}$. At the beginning of each trial run, participants were 
given 5 seconds of driving at a constant speed (40 kph) 18 meters behind the constant speed LV to establish a perception of the desired distance to be maintained. The three sinusoids were out of phase with one another. The initial phase of the high and middle frequency wave was selected randomly with the phase value of the low frequency wave selected to produce a sum on the first frame of zero. This ensured that the beginning of the speed variation of the lead vehicle (following the $5 \mathrm{sec}$ of constant speed) would always be $40 \mathrm{kph}$, yet the velocity profile of the lead car would vary from trial to trial. Located above the roadway was a horizontal array of 21 red and green lights. As the driver approached the lights a single light would change color (either red to green or green to red).

Procedure. Drivers were seated in the simulator and told to perform two tasks: maintain their initial separation from the lead vehicle despite changes in velocity of the lead vehicle and detect, as soon as possible, whether a light change occurred on the left or right side. At the beginning of each trial run, participants were given 5 seconds of driving at a constant speed 18 meters behind the constant speed lead vehicle to establish a perception of the desired distance to be maintained followed by 60 seconds of variations in lead vehicle speed according to the sum of sines function. Light changes occurred every $12 \mathrm{sec}+/-2 \mathrm{sec}$ to prevent drivers from anticipating the light change. Five light change events occurred on every trial. Drivers were given 2 sessions of trials with 2 replications of each distance level presented in a random order. Drivers were given a break between sessions. At the beginning of each session, subjects were given 5 minutes of practice driving to familiarize themselves with the control characteristics of the accelerator and brake.

Drivers were presented with 2 displays in which forward motion was perturbed by single sine waves to illustrate the displays. Once the subjects understood the task they were given two 60sec practice trials in which they responded to the single sine wave forcing function to familiarize the subject with the task and the control dynamics.

\section{Results}

The reaction time and accuracy for the light change detection task is shown in Figure 2. No significant differences were found $(\mathrm{p}>.05)$ for the left/right lcoation of the light change with accuracy and reaction time measures. As a result, other other analyses were collapsed across this variable. The mean accuracy and RT for each driver in each condition was calculated and analyzed in a 3 (horizontal position) by 4 (distance location) analysis of variance (ANOVA). With regard to accuracy, the main effect of light position was significant $(F(2,10)=11.9, p<.05)$. Mean percent correct for the 3, 6 and 9 position conditions were $92.5 \%, 91.4 \%$, and $84.5 \%$, respectively, indicating that accuracy decreased with more peripheral light positions. The main effect of array distance was also significant $(\mathrm{F}(3,15)=5.7, \mathrm{p}<.05)$. Mean percent correct for the $48,72,96$, and $120 \mathrm{~m}$ distance conditions were $91.5 \%, 95.0 \%, 89.5 \%$, and $81.9 \%$, respectively, indicating reduced accuracy with increased distance. An ANOVA of RT results indicated a similar pattern of results. Main effect of light position was significant, $F(2,10)=13.7, p<.05$. with greater RT as a function of more peripheral light positions. With regard to the reaction time results, the main effect of light position was significant, $\mathrm{F}(2,10)=13.8, \mathrm{p}<.05$. Mean $\mathrm{RT}$ was 839 , 938, and 1,160 msec for the 3, 6 and 9 light positions, respectively. The main effect of distance was significant, $\mathrm{F}(3,15)=33.6, \mathrm{p}<.05$. Mean RT was 543, 649, 959, and 1,770 ms for the 48, 72, 96 , and $120 \mathrm{~m}$ conditions. These results indicate that RT increased as a function of distance. 

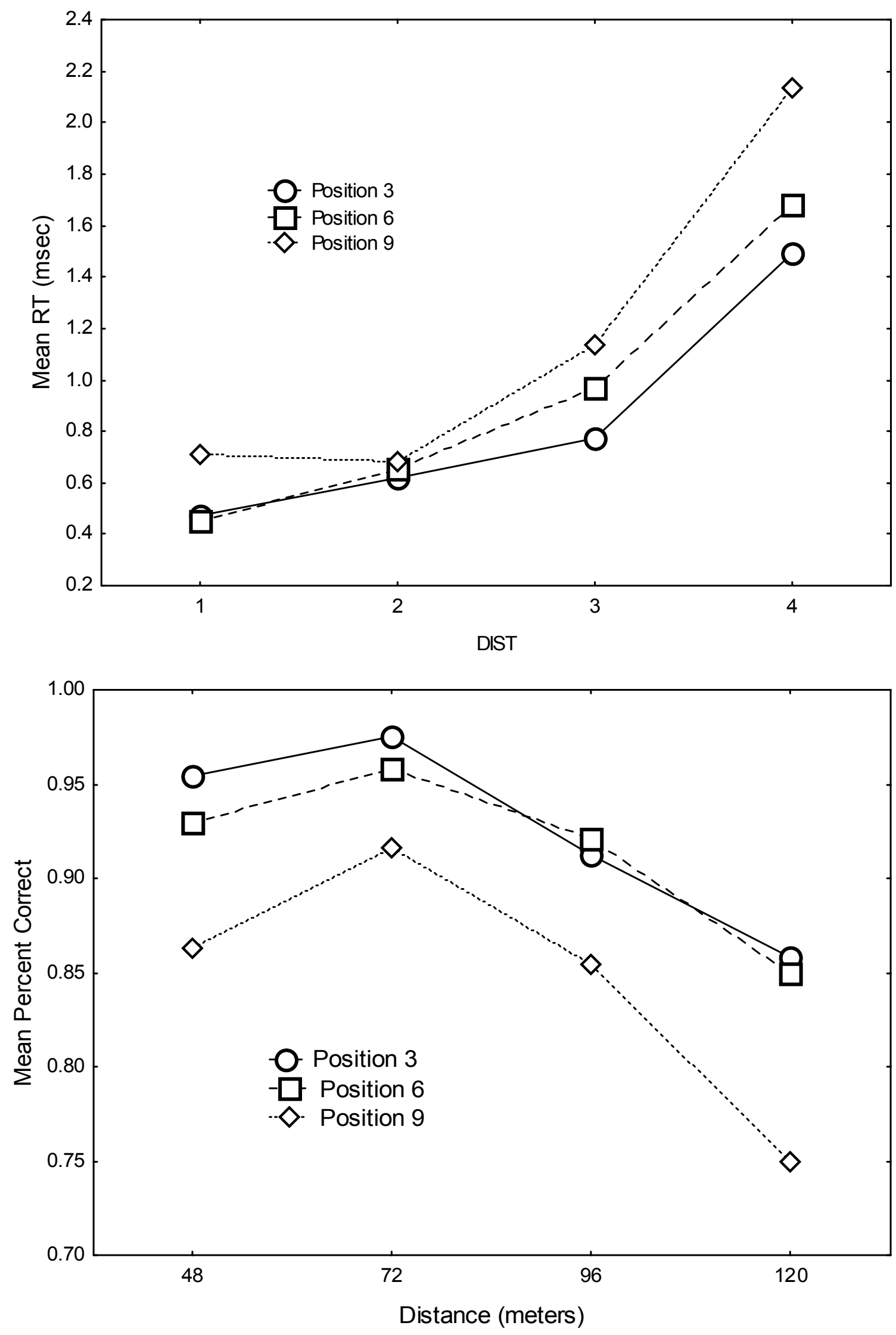

Figure 2. Mean reaction time (top graph) and accuracy (bottom graph) for the light detection change task. 
The two-way interaction of position and distance was significant, $F(6,30)=6.9, p<.05$. According to this result, RT increased with more peripheral light locations, but the difference in RT between central and peripheral light locations was greater at far distances as compared to near distances.

An ANOVA was conducted on car following performance by measuring maintained distance before and after the response to a light change. We found a significant effect of car following performance before and after the response $(\mathrm{F}(1,5)=7.9, \mathrm{p}<.05)$, with a reduction in car following distance after drivers responded to the light change.

\section{Discussion}

The results indicate decreased performance for the secondary task in peripheral vision - a result consistent with previous useful field of view studies as well as studies examining the spatial limitations of visual attention (e.g., Eriksen and Yeh, 1985; Laberge, 1983). However, we found lower accuracy and long RT for detecting light changes positioned at greater distances. As distance increased, the spatial separation between the centrally located lead vehicle and the changing light was decreasd. Thus, light location became more centrally located with increased distance. Measures such as the useful field of view predict that more centrally located peripheral targets are processed more quickly (i.e., the items are located in central regions of the attentional focus). The present findings found the opposite pattern of results - more centrally located targets that were more distant had lower accuracy and longer RT. These results suggest that attending to information in driving is more complex than assumed by measures of attention that are restricted to visual angle.

Of course, one factor that might account for these results is the the size of the target. As distance is increased, the size of the light decreased. Thus, the greater RT and lower accuracy obtained with increased distance may be due to the target item having a smaller visual angle. To determine the relative effects of target distance and target size, we used regression analyses and found that target distance accounted for a higher percentage of performance (accuracy) variability (36\%) than target size (1.2\%). These results suggest that the spatial extent of attention of the driver varied as a function of target distance in the driving scene and was not the effect of a decrease in target size due to increased distance.

\section{ACKNOWLEDGMENTS}

This research was supported by NIA grant NIH AG13419-06 and contract 65A0162 from the California Department of Transportation.

\section{REFERENCES}

Andersen, G. J. (1990). Focused attention in three-dimensional space. Perception \& Psychophysics, 47: 112-120.

Andersen, G. J., \& Kramer, A. F. (1993). Limits of focused attention in three-dimensional space. Perception \& Psychophysics, 53: 658-667. 
Atchley, P., Kramer, A. F., Andersen, G. J., \& Theeuwes, J. (1997). Spatial cuing in a stereoscopic display: evidence for a depth aware attentional focus. Psychonomic Bulletin \& Review, 4: 524-529.

Ball, K. \& Owsley, C. (1993). The useful field of view test: A new technique for evaluating agerelated declines in visual function. Journal of the American Optometric Association, 64: 7179 .

Eriksen, C. W., \& Yeh, Y. Y. (1985). Allocation of attention in the visual field. Journal of Experimental Psychology: Human Perception and Performance, 11: 583-597.

Laberge, D. (1983). Spatial extent of attention to letters and words. Journal of Experimental Psychology: Human Perception and Performance: 371-379.

Sims, R. V., Mcgwin, G., Allman, R. M., Ball, K., \& Owsley, C. (2000). Exploratory study of incident vehicle crashes among older drivers. Journal of Gerontology: Series A, 55: 22-27.

Owsley, C., Ball, K., Sloane, M. E., Roenker, D. L. \& Bruni, J. R. (1991). Visual/cognitive correlates of vehicle accidents in older drivers. Psychology \& Aging, 6: 403-414.

Owsley, C., Ball, K., McGwin, G. Jr. \& et al. (1998). Visual processing impairment and risk of motor vehicle crash among older adults. JAMA: Journal of the American Medical Association. 1998, 279: 1083-1088. 